\title{
Editorial
}

\section{El rol de la simulación en la practica y entrenamiento urológico}

\author{
Herney Andrés García-Perdomo, MD, MSc, EdD, PhD, FACS ${ }^{1}$ Fernando Echeverría-García, MD ${ }^{1}$ \\ Andrés Felipe Gutiérrez Rojas, MD, MSc ${ }^{2}$ \\ ${ }^{1}$ Departamento de Cirugía/Urología. Grupo de Investigación UROGIV. \\ Escuela de Medicina. Universidad del Valle. Cali, Colombia \\ 2 Hospital Universitario San Ignacio, Bogotá DC, Colombia \\ Urol Colomb 2021;30(3):e155-e156.
}

"Yo no temo al hombre que ha lanzado 10,000 patadas diferentes, yo temo al hombre que ha lanzado una patada 10,000 veces" - Bruce Lee

La práctica de la urología ya no es como previamente se concebía. Hace muchos años se pensaba que el modelo de aprendizaje quirúrgico era "lea, vea, ayude, haga y enseñe"; estrategia que permitió formar a grandes, talentosos y hábiles cirujanos, ${ }^{1}$ sin embargo, ya no es de esta manera.

La cirugía mínimamente invasiva y la robótica ha permitido reconocer que se requiere una curva de aprendizaje previo a operar un paciente real. De aquí que un número importante de "horas de vuelo" sean necesarias para adquirir un grupo de habilidades y destrezas para la competencia en un área. ${ }^{2}$ De igual manera, la cirugía es una profesión que depende de la adquisición y aplicación de habilidades psicomotoras, cuya limitación de obtenerlas, se traducirá en consecuencias negativas para su entrenamiento y finalmente para el paciente. ${ }^{3}$

En la actualidad, esta competencia es difícil de alcanzar dada la limitación en el número de horas de actividad asistencial, la legislación actual en nuestro país, la ética, así como limitaciones financieras y administrativas en las organizaciones de salud. ${ }^{4}$ Por tal motivo, se han desarrollado múltiples herramientas de simulación efectiva y segura, en ambientes controlados, por fuera de la sala de operaciones, permitiendo al estudiante y al profesional, adquirir las habilidades necesarias sin comprometer la vida del paciente y la actividad de las organizaciones. ${ }^{2,5}$ Es así, que desde la década del 2000 se han incrementado de manera significativa las publicaciones acerca del entrenamiento en cirugía mínimamente invasiva en urología, particularmente en Estados Unidos y Europa ${ }^{6}$

La simulación es definida como una técnica para “reemplazar o amplificar experiencias reales con experiencias guiadas que evoquen o repliquen aspectos sustanciales de la vida real en una manera totalmente interactiva" ${ }^{5,7}$. La simulación quirúrgica, ha tenido un crecimiento exponencial en la última década, con múltiples estudios fundamentando la necesidad de esta herramienta previo a la realización de procedimientos al paciente. Se han desarrollado estructuras y estudios de entrenamiento basado en simulación, predominantemente observacionales, aunque algunos de ellos con base en modelos experimentales, llevando a evaluar la transferibilidad del conocimiento en los estudiantes y profesionales que inician esta travesía. ${ }^{2}$

Estudios previos han demostrado cómo el entrenamiento mediante el uso de la realidad virtual en cirugía laparoscópica disminuye significativamente el tiempo para completar una tarea, mejora la precisión en la realización de la misma, disminuye los errores y es más efectiva que el entrenamiento mediante el uso de video. De igual forma, se ha demostrado en estudios experimentales como en personas con poco entrenamiento en laparoscopia, la realidad virtual disminuye el tiempo quirúrgico y mejora la economía de movimientos en comparación con el entrenamiento estándar. ${ }^{8}$

Con el advenimiento de la cirugía mínimamente invasiva y la robótica, es importante definir como incorporar la simulación en la educación quirúrgica. Por tanto, vale la pena comprender que la simulación no es un reemplazo del entrenamiento clínico/quirúrgico, por el contrario, es un complemento adjunto a este. Por tanto, la simulación debe ser incorporada en un currículo basado en competencias, que permita que las pocas horas de entrenamiento sean lo mas efectivas posibles. De igual manera, para obtener lo mejor de esta herramienta, el estudiante debe tener una retroalimentación permanente acerca de su desempeño. ${ }^{1}$

Existen múltiples simuladores en ésta tan importante área de la urología, iniciando por lo básico de laparoscopia general, como las cajas de baja fidelidad para aprender movimientos, desempeño y sutura, pasando por el
Address for correspondence Herney Andrés García-Perdomo, $M D, M S c, E d D, P h D, F A C S$, Departamento de Cirugía/ Urología, Grupo de Investigación UROGIV, Escuela de Medicina, Universidad del Valle, Cali, Colombia

(e-mail: herney.garcia@ correounivalle.edu.co).
DOI https://doi.org/ 10.1055/s-0041-1736204. ISSN 0120-789X. e ISSN 2027-0119. (c) 2021. Sociedad Colombiana de Urología. All rights reserved. This is an open access article published by Thieme under the terms of the Creative Commons Attribution-NonDerivative-NonCommercial-License, permitting copying and reproduction so long as the original work is given appropriate credit. Contents may not be used for commercial purposes, or adapted, remixed, transformed or built upon. (https://creativecommons.org/ licenses/by-nc-nd/4.0/)

Thieme Revinter Publicações Ltda., Rua do Matoso 170, Rio de Janeiro, RJ, CEP 20270-135, Brazil 
entrenamiento con simuladores de realidad virtual que mejoran el desempeño en el sistema robótico Da Vinci. ${ }^{5}$

Existen incluso simuladores de alta fidelidad que permiten enseñar habilidades no técnicas, tales como liderazgo, comunicación y trabajo en equipo, como un excelente complemento a las habilidades quirúrgicas técnicas. Finalmente se reflejará en un magnifico trabajo con y para el paciente. ${ }^{9}$

Es de resaltar, que el entrenamiento en simuladores requiere múltiples recursos, así como un grupo de profesores altamente capacitados para ofrecer la mayor efectividad de estas herramientas. Además, aún falta consenso acerca de la validación de las mismas; aunque existen estudios, las aproximaciones son diferentes y aún carecen de estandarización. Es claro que las curvas de aprendizaje para todos los procedimientos son diferentes ${ }^{10}$ y que existen procedimientos muy poco frecuentes con largas curvas, que no se alcanzaran rápidamente. En estos casos, la simulación ofrece una gran ventaja, en asocio con el entrenamiento clínico/quirúrgico, incrementando así las "horas de vuelo"1. Aunque aún faltan estudios con rigor metodológico que permitan evaluar la validez predictiva de éstos, son herramientas que apoyan el entrenamiento y la educación en urología; además, vinieron para quedarse.

Para concluir, la urología es una disciplina con un amplio y continuo uso de tecnología. La utilización de simuladores de baja y alta fidelidad permite al estudiante adquirir las competencias necesarias para la atención de pacientes, disminuyendo así la probabilidad de daño. Es una estrategia que, aunque costosa, nos ayuda a sobrellevar las limitaciones actuales de nuestro sistema educativo y de salud, permitiendo la formación de cirujanos altamente competentes.

\section{Conflict of Interest}

None.

\section{Referencias}

1 Preece R. The current role of simulation in urological training. Cent European J Urol 2015;68(02):207-211

2 Aydin A, Ahmed K, Van Hemelrijck M, Ahmed HU, Khan MS, Dasgupta PSIMULATE Trial Group. Simulation in Urological Training and Education (SIMULATE): Protocol and curriculum development of the first multicentre international randomized controlled trial assessing the transferability of simulation-based surgical training. BJU Int 2020;126(01):202-211

3 Aydin A, Raison N, Khan MS, Dasgupta P, Ahmed K. Simulationbased training and assessment in urological surgery. Nat Rev Urol 2016;13(09):503-519https://pubmed.ncbi.nlm.nih.gov/ 27549358/ cited 2021Aug24 [Internet]

4 Reznick RK, MacRae H. Teaching surgical skills-changes in the wind. N Engl J Med 2006;355(25):2664-2669https://pubmed. ncbi.nlm.nih.gov/17182991/ cited 2021Aug24 [Internet]

5 Kozan AA, Chan LH, Biyani CS. Current status of simulation training in urology: A non-systematic review. Res Rep Urol 2020;12:111-128

6 Torres L, Pedraza A, Arroyo L, et al. Análisis bibliométrico y de mapeo de la educación en cirugía laparoscópica y robótica en urología. Urol Colomb 2020;29(04):202-208. Available from: http://www.thieme-connect.com/products/ejournals/html/ 10.1055/s-0040-1721331

7 Gaba DM. The future vision of simulation in healthcare. Simul Healthc 2007;2(02):126-135

8 Gurusamy K, Aggarwal R, Palanivelu L, Davidson BR. Systematic review of randomized controlled trials on the effectiveness of virtual reality training for laparoscopic surgery. Br J Surg 2008;95 (09):1088-1097https://pubmed.ncbi.nlm.nih.gov/18690637/ cited 2021Aug26 [Internet]

9 Arora S, Lamb B, Undre S, Kneebone R, Darzi A, Sevdalis N. Framework for incorporating simulation into urology training. BJU Int 2011;107(05):806-810https://pubmed.ncbi.nlm.nih.gov/ 20868386/ cited 2021Aug24 [Internet]

10 Grantcharov TP, Bardram L, Funch-Jensen P, Rosenberg J. Learning curves and impact of previous operative experience on performance on a virtual reality simulator to test laparoscopic surgical skills. Am J Surg 2003;185(02):146-149https://pubmed.ncbi. nlm.nih.gov/12559445/ cited 2021Aug26 [Internet] 